BNL-64886-98/10-Rev.

\title{
BEAM INDUCED RF CAVITY TRANSIENT VOLTAGE
}

\author{
S.L. Kramer and Jiunn-Ming Wang \\ National Synchrotron Light Source \\ Brookhaven National Laboratory \\ Upton, New York, USA
}

October 1998

\author{
National Synchrotron Light Source \\ Brookhaven National Laboratory \\ Operated by \\ Brookhaven Science Associates \\ Upton, NY 11973
}

\section{Under Contract with the United States Department of Energy \\ Contract Number DE-AC02-98CH10886}




\section{DISCLAIMER}

This report was prepared as an account of work sponsored by an agency of the United States Government. Neither the United States Government nor any agency thereof, nor any of their employees, nor any of their contractors, subcontractors or their employees, makes any warranty, express or implied, or assumes any legal liability or responsibility for the accuracy, completeness, or any third party's use or the results of such use of any information, apparatus, product, or process disclosed, or represents that its use would not infringe privately owned rights. Reference herein to any specific commercial product, process, or service by trade name, trademark, manufacturer, or otherwise, does not necessarily constitute or imply its endorsement, recommendation, or favoring by the United States Government or any agency thereof or its contractors or subcontractors. The views and opinions of authors expressed herein do not necessarily state or reflect those of the United States Government or any agency thereof. 


\title{
BEAM INDUCED RF CAVITY TRANSIENT VOLTAGE \\ S. L. Kramer and Jiunn-Ming Wang \\ Brookhaven National Laboratory, Upton, NY 11973, USA \\ October 29, 1998
}

\begin{abstract}
We calculate the transient voltage induced in a radio frequency cavity by the injection of a relativistic bunched beam into a circular accelerator. A simplified model of the beam induced voltage, using a single tone current signal, is generated and compared with the voltage induced by a more realistic model of a point-like bunched beam. The high $Q$ limit of the bunched beam model is shown to be related simply to the simplified model. Both models are shown to induce voltages at the resonant frequency $\omega_{r}$ of the cavity and at an integer multiple of the bunch revolution frequency (i.e. the accelerating frequency for powered cavity operation) $h \omega_{o}$. The presence of two nearby frequencies in the cavity leads to a modulation of the carrier wave $\exp \left(j h \omega_{0} t\right)$. A special emphasis is placed in this paper on studying the modulation function. These models prove useful for computing the transient voltage induced in superconducting rf cavities, which was the motivation behind this research. The modulation of the transient cavity voltage discussed in this paper is the physical basis of the recently observed and explained new kind of longitudinal rigid dipole mode which differs from the conventional Robinson mode.
\end{abstract}

\section{Introduction}

Accelerators with high injected beam currents will have large transient voltages induced in their radio frequency $(\mathrm{RF})$ cavities during injection, but most beam loading calculations ignore the transient response and look only at the steady state solution to the beam loading. For superconducting cavities, there has been concern over whether the high transient surface fields could drive the surface into a normal conducting state and quench the RF field. This work was started when we were investigating the feasibility of operating the Superconducting Xray Lithography Source (SXLS) as a coherent light source [1] using a superconducting RF cavity operated with relatively low RF generator power at very large cavity detuning. In addition to the concern for superconducting cavities, the transient beam loading is also of interest for the stability of servo systems during injection with high beam currents.

This paper will deal primarily with the transient beam loading in RF cavities for relativistic bunched beams. The next section will layout the basic equations for calculating the beam induced voltages in a resonant cavity, the third section will calculate the transient voltage for a simplified switched on single tone current and the fourth section will calculate the corresponding voltage for periodic transits of a bunched beam through a resonant cavity. The high Q limit of the results of this section will be calculated in the next section and compared with the simplified miodel of section three. 


\section{Wakefield and Impedance}

The RF cavity voltage induced by the beam current $I(t)$ is

$$
V(t)=-\int_{-\infty}^{\infty} d t^{\prime} W\left(t-t^{\prime}\right) I\left(t^{\prime}\right)
$$

where $W(t)$ is the wake function. For a very relativistic beam, the wake function for a resonant cavity, with resonant frequency $\omega_{r}$ and quality factor $Q$ is [2]

$$
W(t)=\left\{\begin{array}{ll}
2 k_{\circ} \cos \omega_{r} t e^{-\Gamma t} & \text { if } t>0 \\
k_{\circ} & \text { if } t=0 \\
0 & \text { if } t<0
\end{array},\right.
$$

where the loss factor $k_{o}=R_{s h} \Gamma$, with $R_{s h}=$ shunt impedance, and $\Gamma=\omega_{r} / 2 Q$. The quantity $T_{f} \equiv 1 / \Gamma$ will be refered to as the filling time. The equality $W(0)=W(0+) / 2$ is the wellknown "fundamental theorem of beam loading" [3] which states that the bunch sees only half of the voltage the bunch itself induces in the same passage. In terms of the complex resonance frequency $\Omega \equiv \omega_{r}+j \Gamma$, the beam impedance corresponding to the above wake function is

$$
\begin{aligned}
Z(\omega) & =\int_{-\infty}^{\infty} d t W(t) e^{-j \omega t} \\
& =-j k_{o}\left(\frac{1}{\omega-\Omega}+\frac{1}{\omega+\Omega^{*}}\right) .
\end{aligned}
$$

The damping coefficient $\Gamma$ of the wake function can be easily seen to be half of the bandwidth of the impedance in Eq.(4).

It is convenient to define the impedance function

$$
\zeta(\omega)=\frac{-j 2 k_{\circ}}{\omega-\Omega},
$$

and the corresponding complex wake function

$$
U(t)=\frac{1}{2 \pi} \int_{-\infty}^{\infty} d \omega e^{j \omega t} \zeta(\omega)=\left\{\begin{array}{ll}
2 k_{o} e^{j \Omega t} & \text { if } t>0 \\
k_{o} & \text { if } t=0 \\
0 & \text { if } t<0
\end{array} .\right.
$$

Then the actual cavity gap voltage $V(t)$ is given by the real part of the complex voltage $\nu(t)$

$$
V(t) \equiv \operatorname{Re}[\nu(t)]=\operatorname{Re}\left[-\int_{-\infty}^{\infty} d t^{\prime} U\left(t-t^{\prime}\right) I\left(t^{\prime}\right)\right]
$$

In terms of the frequency detune angle $\Theta$ defined by 


$$
\Delta \equiv \omega-\omega_{r}=\Gamma \tan \Theta,
$$

the impedance function then can be expressed by

$$
\zeta(\omega)=2 R_{s h} \cos \Theta e^{-j \Theta} .
$$

These relations will be used below to express the transient cavity voltage induced by the beam.

\section{$3 \quad$ A Simplified Model}

We first consider a very simple example where the beam current is given by a switched on single tone signal at $t=0$ given by

$$
I(t)=\left\{\begin{array}{ll}
2 I_{a v} \cos h \omega_{o} t, & \text { if } t>0 \\
0, & \text { if } t<0
\end{array} .\right.
$$

In Section 5 , the voltage induced in a high $\mathrm{Q}$ cavity by the periodic passage of a bunched beam of charge $q$ at $t=0, T_{o}, 2 T_{o}, \ldots$, where $T_{o}=2 \pi / \omega_{0}$ and $I_{a v}=q / T_{o}$, will be shown to be closely related to that induced by the current in Eq.(10).

The complex cavity voltage induced by the current in Eq.(10) can be written in a phasor form corresponding to the operating (accelerating) frequency $h \omega_{0}$. From Eqs.(7) and (10), the voltage is given by

$$
\begin{gathered}
\nu_{s m l}(t)=\tilde{V}_{s m}(t) e^{j h \omega_{Q} t}, \quad \text { with } \\
\tilde{V}_{s m}(t) \cong-2 I_{a v} R_{s h} \cos \Theta e^{-j \Theta}\left[1-e^{-j \Delta t} e^{-\Gamma t}\right],
\end{gathered}
$$

where the detune frequency $\Delta$ is given by Eq.(8) and the subscript $s m$ is used to indicate "Simplified Model". The correction to the approximation in Eq.(12) is smaller by a factor of $O(1 / 2 Q)$. The time variation of the modulation function $\tilde{V}_{s m}$ given by Eq.(12) is determined by the two parameters $\Delta$ and $\Gamma$.

The first term on RHS of Eq.(12), which gives a constant amplitude to the term with frequency $h \omega_{o}$ in Eq.(11), is the asymptotic solution to the induced voltage (i.e. steady state solution). The last term is the transient voltage induced by the discontinuity in the current at $t=0$. This discontinuity can be considered to provide an $\infty$ bandwidth to the current signal and can therefore excite all frequencies of the resonance, Eq.(5). As a consequence, the transient voltage gives a term to Eq.(11) which oscillates with the resonant frequency $\omega_{r}$ instead of the driving frequency $h \omega_{0}$. 

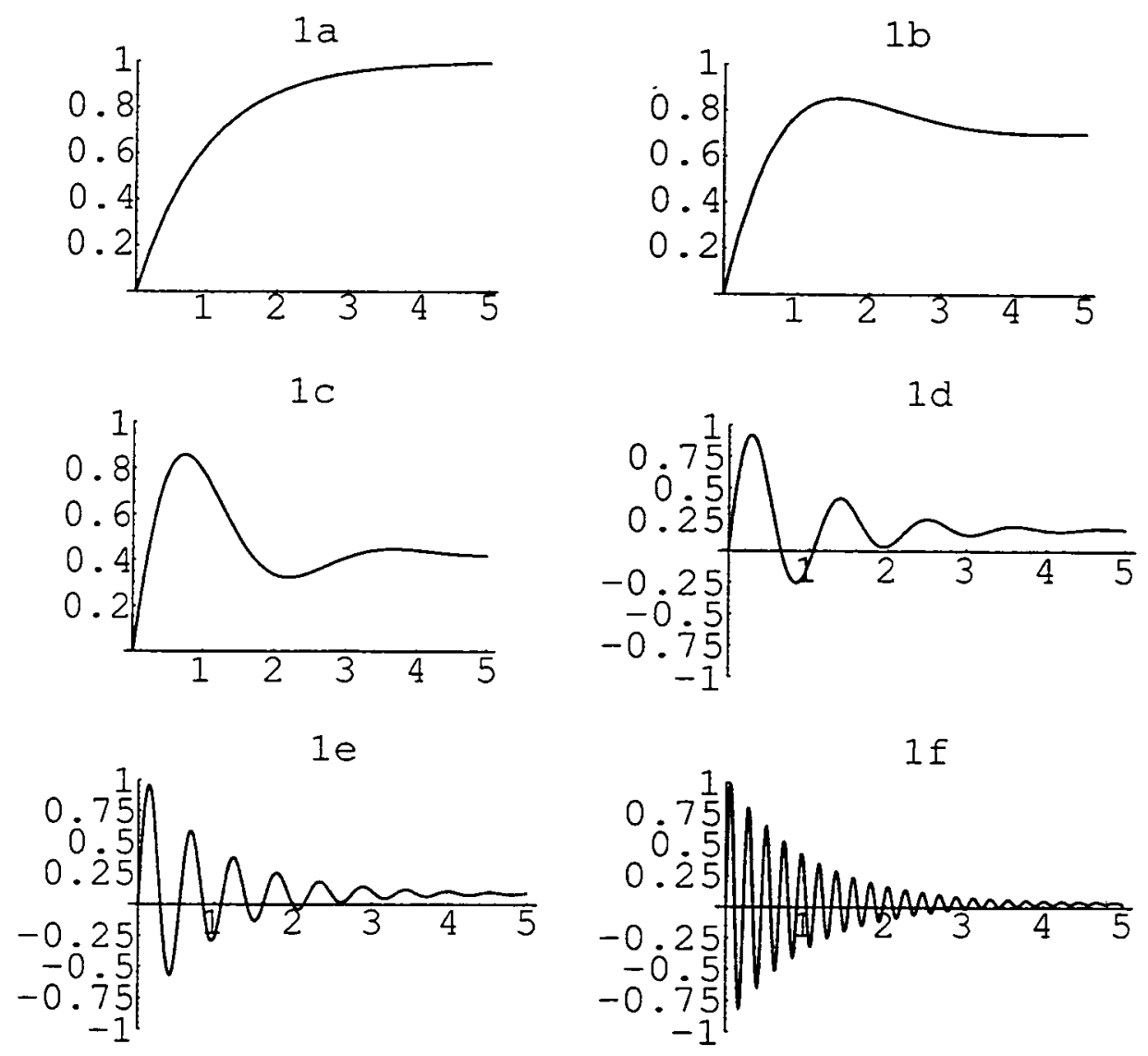

Fig. 1: The sampled transient voltage, Eq.(14), for the Simplified Model. The horizontal axes are $\bar{t}=t / T_{f}$ and the voltage on the vertical axes are in arbitrary units. Graphs from la to lf are, respectively, for $\Theta=0^{\circ}, 45^{\circ}, 65^{\circ}, 80^{\circ}, 85^{\circ}$ and $88^{\circ}$.

In order to visualize the envelope function $\operatorname{Real}\left[\tilde{V}_{s m}\right]$, it is convenient to sample the voltage at $t=t_{p} \equiv p T_{0}$, with $p=0,1,2 \ldots$, so that the carrier wave $\exp \left(j h \omega_{0} t\right)=1$. From Eqs.(11) and (12),

$$
\begin{aligned}
\nu_{s m}\left(t_{p}\right) & =\tilde{V}_{s m}\left(t_{p}\right), \\
& =-2 I_{a v} R_{s h} \cos \Theta e^{-j \Theta}\left[1-e^{-j \Delta t_{p}} e^{-\Gamma t_{p}}\right] .
\end{aligned}
$$

The cavity voltage at these instants are given by the real part of Eq.(13). Let us define a dimensionless quantity $\bar{t} \equiv t_{p} / T_{f}$; it is the sampled time expressed in units of the filling time $T_{f}$. Then the real part of the above equation can be written as

$$
V_{s m}(\bar{t}, \Theta)=-2 I_{a v} R_{s h} \cos \Theta[\cos \Theta-\cos (\bar{t} \tan \Theta+\Theta) \exp (-\bar{t})]
$$

Note that in this expression the explicit dependence of $V_{s m}$ on $\Gamma$ is "scaled out". The sampled cavity voltage as given by Eq.(14) is plotted for various detune angles in Fig. 1.

As a measure of the magnitude of the transient-voltage fluctuation, let us define for a given $\Theta$

$$
\xi(\Theta)=\left[\operatorname{maximum}\left(\left|V_{s m}\right|\right)\right] /\left|V_{s m}(\bar{t} \rightarrow \infty)\right| .
$$


From Fig. 1, it is clear that the absolute maximum of $\left|V_{s m}\right|$ is the local maximum corresponding to the smallest $\bar{t}$. Simple arithmatics shows that the maximum occurs at $\bar{t}=\pi / 2 \tan \Theta$, and

$$
\xi(\Theta)=1+\tan \Theta \exp (-\pi / 2 \tan \Theta) .
$$

The parameter $\xi$ as plotted in Fig.2 increases slowly when $\Theta$ increses from 0 to $\sim 70^{\circ}$. However it starts to increase rapidly around $\Theta \sim 80^{\circ}$.

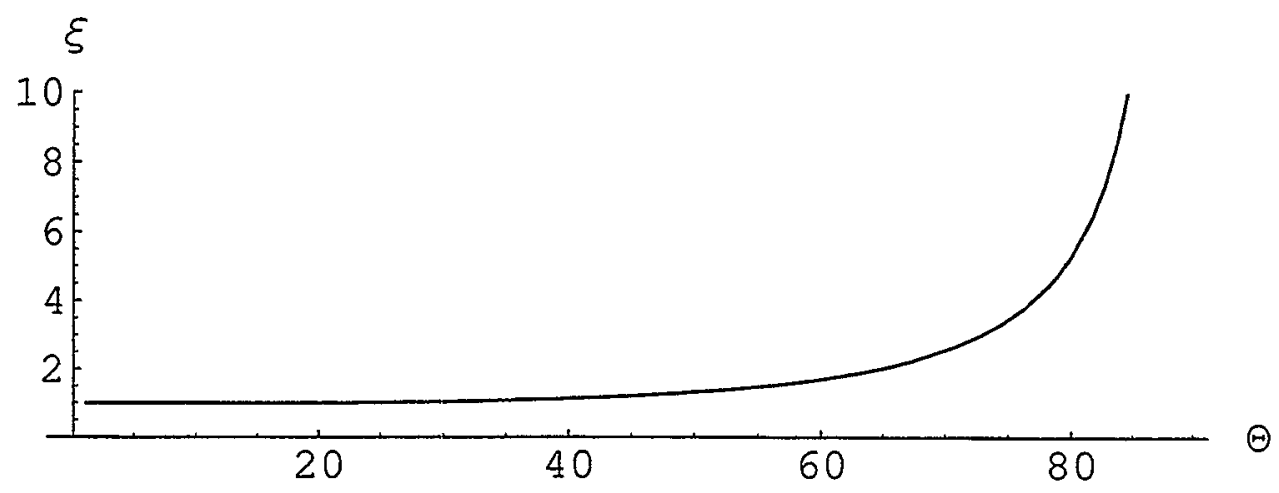

Fig. 2: The transient-voltage-fluctuation parameter $\xi$ as a function of the detuning angle $\Theta$.

We summarize this section by noting that the voltage $V_{s m}$ as depicted in Fig. 1 satisfies the following conditions: (a) it vanishes at $\bar{t}=0$, and (b) it is a function of only two variables $\bar{t}$ and $\Theta$. We shall see in the next section that these characteristics of the simplified model do not hold true for a more realistic bunched beam model.

\section{Bunched Beam Induced Voltage}

In this section, we consider the case of a point-like bunch of charge $q$ passing the cavity at $t=0, T_{o}, 2 T_{o}, \ldots .$. The beam current seen by the cavity is

$$
I(t)=q \sum_{p=0}^{\infty} \delta\left(t-p T_{0}\right)
$$

The current impulse at $t=p T_{o}$ introduces a discontinuity of the induced voltage at the same instant. To calculate the complex voltage $\nu(t)$ induced by this periodic impulse, we introduce a reduced time $\tau, \tau \equiv t-N_{t} T_{o}$, where $N_{t}$ is the integer part of $t$ and $0<\tau<T_{0}$. The contribution of any $p$-th term, $p \leq N_{t}$, in Eq.(17) to the cavity voltage is, easily calculated from Eq.(7) and the complex.voltage induced by the current in Eq.(17) is given by

$$
\nu(t)=-2 q k_{o} \sum_{p=0}^{N_{t}} \exp \left[j \Omega\left(N_{t} T_{o}+\tau-p T_{o}\right)\right] .
$$


An instructive approach is to express this voltage as the sum of two terms

$$
\nu(t)=\nu_{o}(t)-\nu_{1}(t),
$$

where $\nu_{o}(t)$ and $\nu_{1}(t)$ are the voltages induced, respectively, by the currents

$$
I_{o}(t)=q \sum_{p=-\infty}^{\infty} \delta\left(t-p T_{o}\right)
$$

and

$$
I_{1}(t)=q \sum_{p=-\infty}^{-1} \delta\left(t-p T_{0}\right)
$$

Then the induced voltages for these currents are calculated from Eqs.(6) and (7) and simplified by using the Poisson sum rule to give the expressions:

$$
\nu_{o}(t)=2 k_{\circ} q e^{j \Omega \tau} /\left(e^{j \Omega T_{o}}-1\right)
$$

and

$$
\nu_{1}(t)= \begin{cases}2 k_{o} q e^{j \Omega t} /\left(1-e^{-j \Omega T_{o}}\right) & \text { for } t>0 \\ \nu_{o}(t) & \text { for } t<0\end{cases}
$$

The equilibrium distribution (22) has been obtained previously by Boussard [4]. This function is periodic in $t$ with period $T_{o}$, but with discontinuities at $t=N_{t} T_{o}$. Its Fourier series expansion is given by [5]

$$
\nu_{o}(t)=j I_{a v} \sum_{n=-\infty}^{\infty} \frac{2 k_{o}}{n \omega_{o}-\Omega} e^{j n \omega_{o} t}
$$

where $\omega_{o}=2 \pi / T_{o}$ and $I_{a v}=q / T_{o}$.

The total induced voltage, $\nu(t)$, can be obtained from Eqs.(19), (22) and (23). We see $\nu(t)$ vanishes for $t<0$, and

$$
\nu(t)=2 k_{\circ} q\left[e^{j \Omega \tau}-e^{j \Omega t} e^{j \Omega T_{\circ}}\right] /\left(e^{j \Omega T_{\circ}}-1\right) \quad \text { for } \quad t>0 .
$$

The Fourier expansion of this voltage is given by

$$
\nu(t)=j I_{a v} \sum_{n=-\infty}^{\infty} \frac{2 k_{o}}{n \omega_{o}-\Omega} e^{j n \omega_{o} t}-2 k_{o} q e^{j \Omega t} e^{j \Omega T_{o}} /\left(e^{j \Omega T_{o}}-1\right) .
$$

In the next section, we consider an approximation of this voltage for a high $Q$ resonance. 


\section{High $Q$ Resonance}

In this section we look at the bunched beam induced voltage for a resonance with $Q$ large enough, $\left(\Gamma / \omega_{0} \approx h /(2 Q) \ll 1\right)$, that only one term $n=h$ in Eq.(26) falls within the bandwidth of the resonance. The terms with $n \neq h$ are smaller than this term by $O\left(\Gamma / \omega_{0}\right)$ and can be neglected in Eq.(26). Then by approximating the denominator of the last term of Eq.(26) by

$$
\begin{aligned}
\exp \left(j \Omega T_{o}\right)-1 & =\exp \left[-j\left(h \omega_{0}-\omega_{r}-j \Gamma\right) T_{0}\right]-1 \\
& \cong-j\left(h \omega_{0}-\omega_{r}-j \Gamma\right) T_{0}
\end{aligned}
$$

we obtain for Eq.(26)

$$
\nu(t) \cong \frac{2 j I_{a v} k_{o}}{\Delta-j \Gamma}\left[\exp \left(j h \omega_{o} t\right)-\exp \left(j \omega_{r} t-\Gamma t\right) \exp \left(-j \Delta T_{o}-\Gamma T_{0}\right)\right]
$$

Similar to Eq.(11), this equation can be expressed in terms of a phasor at the operating frequency $h \omega_{0}$ using the detune angle, Eq.(8). The result is

$$
\nu(t)=\tilde{V}_{b b}(t) e^{j \hbar \omega_{0} t},
$$

where the modulation function for bunched beam, subscript $b b$, is given by

$$
\tilde{V}_{b b}(t) \cong-2 I_{a v} R_{s h} \cos \Theta e^{-j \Theta}\left[1-e^{-j \Delta t} e^{-\Gamma t} \exp \left(-\Gamma T_{o} e^{j \Theta} / \cos \Theta\right)\right] .
$$

Comparison of this with the corresponding voltage of the simplified model, Eq.(12), shows that the only difference between these two expressions is the factor $\exp \left(-\Gamma T_{0} e^{j \Theta} / \cos \Theta\right)$ inside the bracket of Eqs.(30). This factor guarantees that

$$
\tilde{V}_{b b} \rightarrow-2 q k_{o} \quad \text { as } \quad t \rightarrow 0+,
$$

as expected. In the last equation, a term smaller by a factor of $O(\gamma)$ where $\gamma \equiv \Gamma T_{0}$ is the cavity decay rate per revolution, is ignored in line with the present approximation.

Sampling this phasor voltage again at $t=t_{p}+\equiv p T_{0}$ in the limit of $\tau \rightarrow 0+$, with $p=0,1,2 \ldots$, and again using the notation $\bar{t} \equiv t_{p} / T_{f}$, we obtain the induced voltage

$$
\begin{aligned}
V_{b b}(\bar{t}, \Theta, \gamma) & =\operatorname{Re}\left[\tilde{V}_{b b}\left(t_{p}+\right)\right] \\
& =-2 I_{a v} R_{s h} \cos \Theta[\cos \Theta-\cos \{(\bar{t}+\gamma) \tan \Theta+\Theta\} \exp (-\bar{t}-\gamma)] .
\end{aligned}
$$

While $V_{s m}$ as given by Eq. (14) was independent of $\Gamma, V_{b b}$ above depends on $\Gamma$ through $\gamma$. However, it is interesting that the two voltages are related simply by

$$
V_{b b}(\bar{t}, \Theta, \gamma)=V_{s m}(\bar{t}+\gamma, \Theta) .
$$

Because of this relation, we can infer the behavior of $V_{b b}$ from that of $V_{s m}$; the plots for $V_{b b}$ is the same as those for $V_{s m}$, Fig. 1, except that the figures should be shifted to the left by $\gamma$. 
Note that this shift in $\bar{t}$ is equivalent to a shift in $t_{p}$ by $T_{o}$. Also note that if we define $\bar{t}=\Gamma t$ in the equations (12) and (30), we obtain a similar relation for the modulation functions themselves

$$
\tilde{V}_{b b}(\bar{t}, \Theta, \gamma)=\tilde{V}_{s m}(\bar{t}+\gamma, \Theta) .
$$

Some important consequenses of this shift in $\bar{t}$ are:

(i) unlike $V_{s m}, V_{b b}$ does not vanish at $\bar{t}=0$; the amount of the shift is so as to reproduce the desired result Eq.(31), and

(iii) the expression (16) for the transient-voltage-fluctuation parameter is still valid for the high $\mathrm{Q}$ bunched beam model.

\section{Conclusions}

It has been shown in this paper that the transient beam loading induces voltages at two frequencies: $h \omega_{o}$ and $\Omega=\omega_{r}+j \Gamma$. It is the beating of these two frequencies that causes the total voltage to have oscillations in its envelope, as shown in Fig. 1. Although these frequencies have been observed during injection, they have not been shown to cause any significant problem for conventional RF cavity systems. However, in the design of a RF system using superconductive cavity with a relatively low generator power and with a high cavity detuning, the transient-voltage fluctuation is bound to become a major constraint.

We end this paper by noting that the presence of two frequencies in the cavity has a very important consequence on the coherent behavior of bunched beams in the storage ring [6]. It leads to a longitudinal rigid dipole beam mode with coherent frequency $\Delta+j \Gamma$ in the limit of vanishing beam current; this mode is distinct from the conventional Robinson mode which $\rightarrow$ the synchrotron frequency in the same beam current limit. This new coherent dipole mode was actually first observed in 1995 experimentally by users on the VUV Ring at the NSLS [7] and it has since been known to degrade the infra-red photon beam unless care is taken in choosing the operating detune angle. 


\section{References}

[1] J.B. Murphy and S. Krinsky, Nuc. Inst. \& Meth. 346A (1994)p.571.

[2] K. Bane, P. Wilson, and T. Weiland, "Wake-Fields and Wake-Field Acceleration", AIP Conference Proceedings No.127 (1985).

[3] P. Wilson, "High Energy Electron Linacs: Applications to Storage Ring RF Systems and Linear Colliders", AIP Conference Proceedings No.87,(1981)p.452. For an elegant and intuitive treatment of this theorem, see Boussard's paper on beam loading cited below.

[4] D. Boussard, "Beam Loading", Proceedings of CERN Accel. School, CERN 87-03, p.626. The discrepancy between Boussard's equation (8) and our equation (22) is due to the fact that Boussard's expression describes the voltage seen by the beam $(\tau=0)$, while our expression is for $\tau>0$. Boussard's expression can be obtained easily from ours by using the fundamental theorem of beam loading.

[5] J.M. Wang and C. Pellegrini, BNL-51236 (1979).

[6] N. Towne and J.M. Wang, "Spectrum of Single Bunch Longitudinal Dipole Modes", Phys. Rev. E 57 (1998) p.3461.

[7] R. Biscardi, et al, Rev. Sci. Instrum. 66 (1995) p.1856. 
